\title{
Graphene oxide and lipid membranes: Size-dependent interactions
}

(Supporting information)

Rickard Frost ${ }^{1,}{ }^{*}$, Sofia Svedhem², Christoph Langhammer ${ }^{2}$, and Bengt Kasemo ${ }^{2, *}$

${ }^{1}$ Department of Energy and Environment, Chalmers University of Technology, SE41296 Göteborg Sweden

2 Department of Applied Physics, Chalmers University of Technology, SE-412 96

Göteborg Sweden

*Corresponding Authors: Rickard Frost, e-mail: rickard.frost@chalmers.se

Bengt Kasemo, e-mail: kasemo@chalmers.se 


\section{Results}

XPS analysis of GO samples

Details regarding the analyses of the XPS data (C1s spectra) are presented in Table

S1.

Table S1. XPS data of GO 1-3

\begin{tabular}{|c|c|c|c|c|}
\hline Peak & $\begin{array}{l}\text { Functional } \\
\text { group }\end{array}$ & Sample & $\begin{array}{c}\text { Binding energy } \\
(\mathrm{eV})^{*}\end{array}$ & $\begin{array}{c}\text { Atomic percent } \\
(\%)^{* *}\end{array}$ \\
\hline \multirow[t]{3}{*}{1} & $\mathrm{O}-\mathrm{C}=\mathrm{O}$ & GO 1 & 288.2 & 9.1 \\
\hline & & GO 2 & 288.0 & 4.4 \\
\hline & & GO 3 & 288.0 & 5.2 \\
\hline \multirow[t]{3}{*}{ II } & $C=O$ & GO 1 & 287.0 & 9.4 \\
\hline & & GO 2 & 286.8 & 6.0 \\
\hline & & GO 3 & 286.8 & 9.1 \\
\hline \multirow[t]{3}{*}{ III } & $\mathrm{C}-\mathrm{O}$ & GO 1 & 285.9 & 40.1 \\
\hline & & GO 2 & 285.6 & 49.7 \\
\hline & & GO 3 & 285.6 & 44.2 \\
\hline \multirow[t]{3}{*}{ IV } & $C-C$ & GO 1 & 283.8 & 41.4 \\
\hline & & GO 2 & 283.6 & 39.8 \\
\hline & & GO 3 & 283.6 & 41.6 \\
\hline
\end{tabular}

\footnotetext{
*The binding energies are shifted about $1 \mathrm{eV}$ towards lower energies compared to literature values.

**Peak $\mathrm{V}$, attributed to hydrocarbon contamination of the sample surface, present in sample GO 2 has been disregarded.
} 
GO adsorption to lipid membranes

Lipid membranes were formed on $\mathrm{SiO}_{2}$ surfaces and then exposed to GO. In Figure S1, a characteristic INPS response of the GO (GO 2) adsorption to a POPC:POEPC (3:1) membrane is shown. Usually, a positive $\Delta \lambda$ is expected upon adsorption due to the increased RI within the penetration depth of the sensor. The observed initial negative $\Delta \lambda$ is likely due to wavelength dependent scattering of GO in suspension.

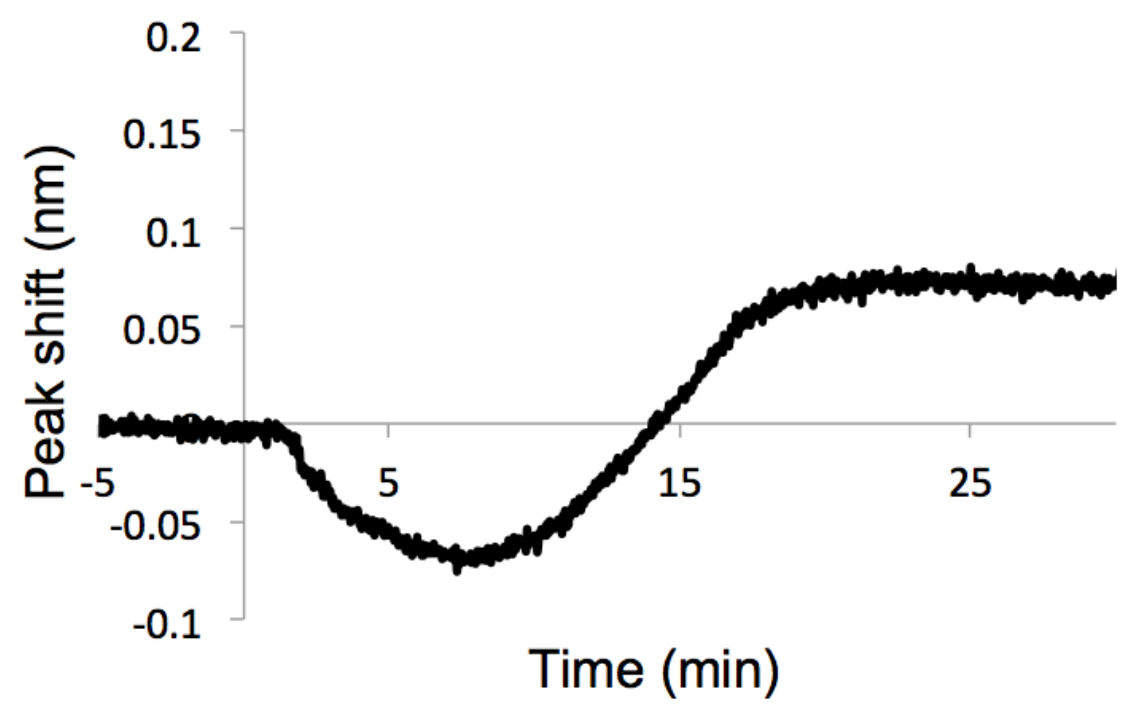

Figure S1. INPS data of GO (GO 2) adsorption to POPC:POEPC (3:1) supported lipid membrane.

Size distributions of SUVs and LUVS

In Figure S2 representative examples of the intensity and number based size distributions are shown for both SUVs and LUVs. The results show that there is a clear difference in size between the two samples. 

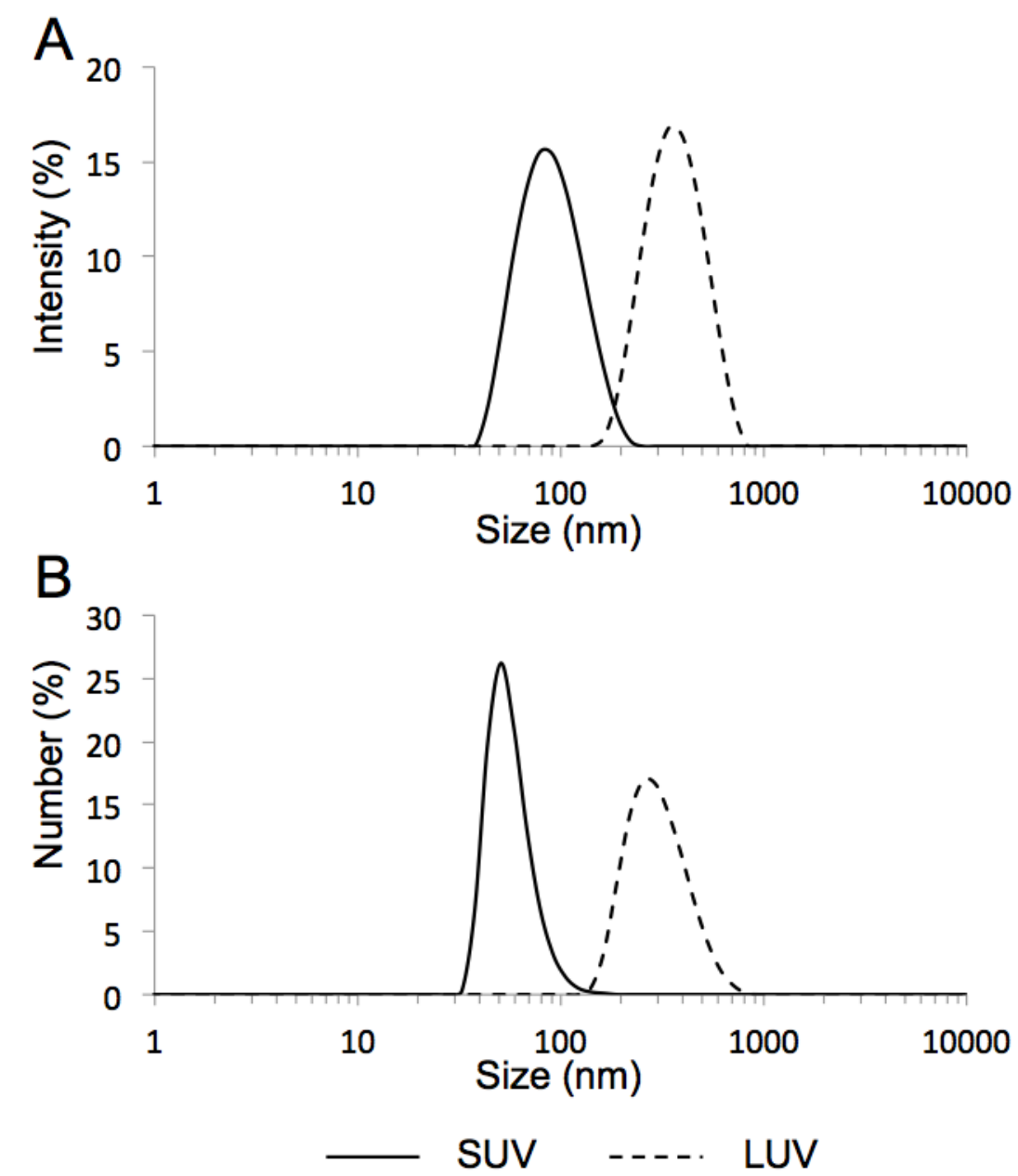

Figure S2. DLS data of the size distributions of SUVs (solid line) and LUVs (dashed line) based on (A) intensity and (B) number.

\section{Formation of supported lipid membranes}

The mechanistic scenario of the supported lipid membrane formation process from extruded vesicles is briefly described in the article. In Figure S3 the QCM-D and INPS responses of this process are shown when using POPC:POEPC (3:1) SUVs and LUVs. It is evident that the final responses are the same, i.e. it is possible to form a supported lipid membrane using both types of liposomes. However, the kinetics of 
the process differs with the size of the liposomes, where LUVs have a slow diffusion rate compared to SUVs. Thus, the overall formation of a lipid membrane occurs more slowly.
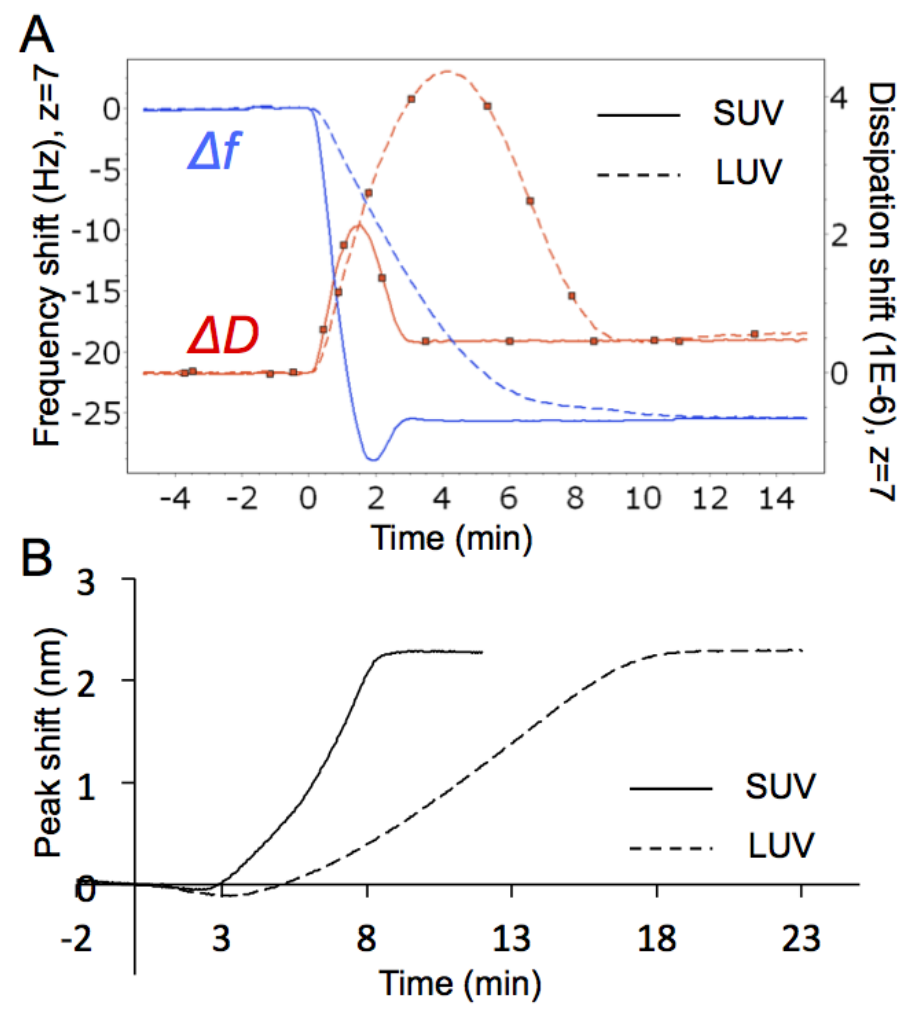

Figure S3. Formation of supported lipid membranes from SUVs (solid line) and LUVs (dashed line) monitored by (A) QCM-D and (B) INPS. 